\title{
Gene delivery from replication-selective viruses: arming guided missiles in the war against cancer
}

\author{
Terry Hermiston
}

Onyx Pharmaceuticals, 3031 Research Drive, Richmond, California 94925, USA.

Phone: (510) 262-8719; Fax: (510) 222-9758; E-mail: thermiston@onyx-pharm.com.

Gene therapy aims to deliver therapeutic genetic material in a safe and efficient manner to a target tissue, where it can accumulate to levels that afford maximal patient benefit. Replication-defective viral vectors have been the workhorse of gene therapy for cancer and other conditions. These vectors allow the efficient delivery of a variety of transgenes to target tissues and have demonstrated clear therapeutic benefit and safety in a variety of preclinical neoplastic animal models. Unfortunately, the tremendous promise of studies using replicationdefective viruses in preclinical models has not been translated into similar patient benefits in the clinical setting (1-3). This shortfall may be due in large part to the one-dimensional nature of these approaches, asking for a successful therapeutic outcome against a highly complex biological target like a human tumor through the activity of a single gene. Therapeutic gene delivery needs to develop into a multidimensional system if it is to be successful in treating human cancers. A natural evolution of gene therapy is its incorporation into replication-selective oncolytic viruses, combining the antitumor properties of the viral infection with the action of the therapeutic proteins.

\section{Oncolytic replication-selective viruses}

For nearly a century, the idea of using replication-competent viruses to treat human cancer has been revisited with various viruses (4). However, only recently has our understanding of viral and cancer biology progressed to a stage where replication-selective tumor-specific viruses could be genetically engineered. To date, the viruses that have been administered in the clinical setting are derivatives of human adenovirus (Ad) (see Heise and Kirn, this Perspective series, ref. 5) and herpes simplex virus (HSV) (see Martuza, this Perspective series, ref. 6). Here, I focus on these two viruses, mindful that many of the principles discussed here will have broad application in other developing biotherapeutic systems.

The treatment of human tumors with a replicationselective, transgene-expressing virus is a natural extension of virus-mediated gene delivery that, in theory, offers several potential advantages. The oncolytic virus is itself capable of lysing the infected tumor cell to eradicate or reduce the tumor mass. Importantly, replication leads to amplification of the input "dose" of the virus, and these progeny viruses are released by virusmediated lysis of the infected cell to spread and infect surrounding cells. This self-perpetuating treatment continues until the host immune response and/or susceptible cells become limiting (Figure 1). Substantial tumor specificity can be achieved by careful genetic engineering of the viral genome to develop viruses that selectively replicate in cancer cells. For example, ONYX015 is a replication-selective Ad lacking a viral gene, $E 1 B-55 K$, whose product is required to inactivate the cellular tumor suppressor p53 (7-10). This virus replicates in and preferentially lyses tumor cells that lack functional p53. An additional benefit of this approach is that virus-mediated lysis of neoplastic cells then frees tumor antigens and should supply dendritic cells and $T$ cells with various signals necessary for immune recognition (11), thus augmenting the development of systemic antitumoral immunity (12).

\section{Advantages of selectively replicating viral systems for gene delivery}

In addition to their oncolytic capabilities, replicating viruses can deliver therapeutic transgenes to enhance the probability of tumor eradication through multiple avenues of attack. As in replication-defective viruses, transgene expression from these replicating agents can be controlled by constitutive, inducible, or cell type-selective transcriptional control elements. Unlike replication-defective viruses, however, replication-selective viral systems can also employ endogenous viral gene expression control signals (promoter/enhancer, polyadenylation, and splice signals) for transgene expression. Eliminating the need for exogenous promoters and polyadenylation signals is an economical use of the limited transgene capacity afforded a replicating viral agent; it is especially advantageous in working with human Ad, whose virion can only stably package up to $105 \%$ of the wild-type viral genome, or approximately 1.8 kilobase pair (kbp) (13). Using endogenous viral promoters may also allow more predictable and controlled transgene expression. Tumor-specific promoters have been used to impose selectivity on replication-selective oncolytic viruses by controlling genes expressed immediately after infection (14), but viruses modify infected cells in many ways to maximize viral replication. How these modifications might affect the exogenous promoters through the course of the viral life cycle is largely unknown. In contrast to foreign or exogenous promoters, the promoters of the replicating agent are optimized for expression in the infected cell.

Viral gene expression during the lytic phase of the viral life cycles of both HSV and Ad is highly regulated and can be broadly classified into three serially activated phases: immediate-early (IE), early (E), and late (L). Based on the expression of endogenous viral genes, it may be possible to predict the expression kinetics (timing and expression levels) of the transgene(s) carried by the replicating agent. Furthermore, when multiple transgenes are inserted into a single 


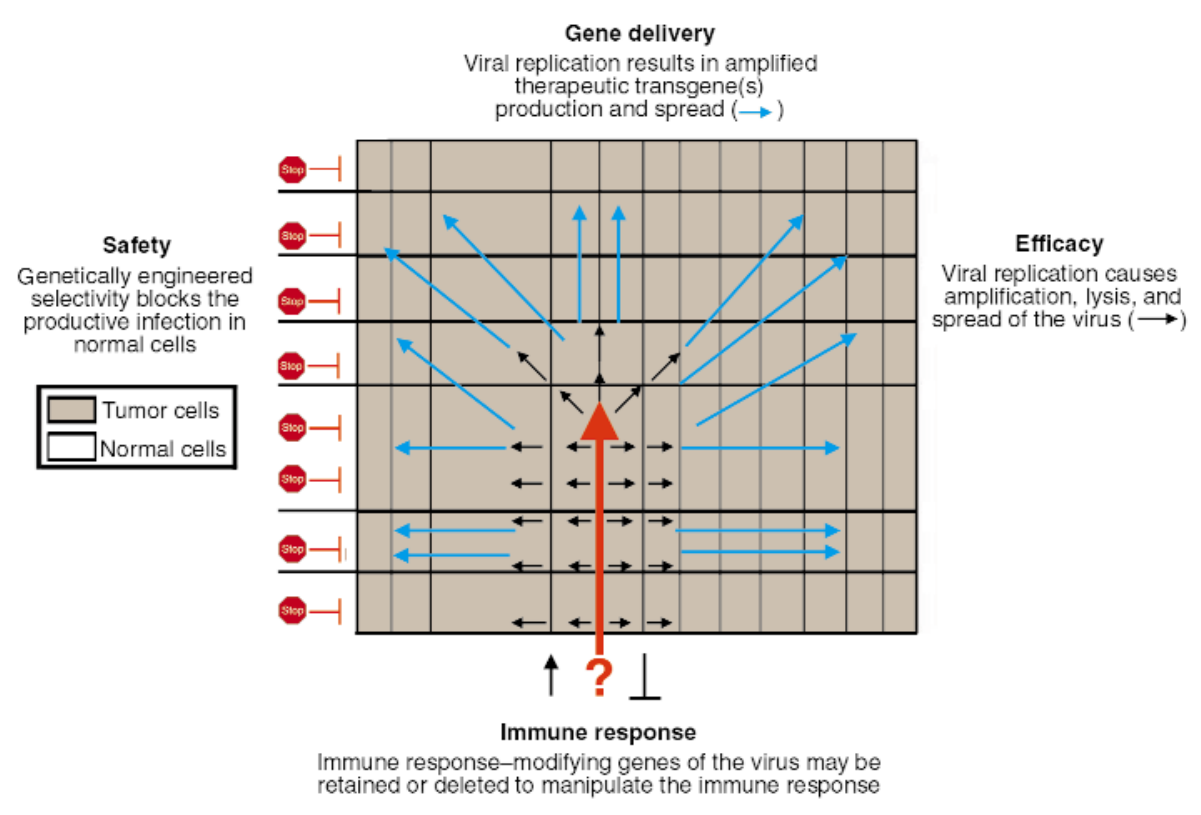

Figure 1

Theoretical advantages of replicationselective, transgene-expressing oncolytic viruses. As several articles in this series show, cytolytic viruses can be engineered that exploit the quirks of cancer cells and spare neighboring tissues. The melding of this technology with gene therapy offers the prospect of safe treatments, enhanced killing of tumor cells, and subtle control over immune responses to tumor cells or viral antigens. virus, their expression may be orchestrated to occur simultaneously or serially, at levels that will maximize their therapeutic benefit. Expressing transgenes serially, at different times in the viral lytic cycle, is of greatest value early in treatment when the infection may be more synchronized. As a viral infection spreads and encounters a heterogeneous tumor cell mass, it will likely become asynchronous, although the relative expression of different transgenes may still be maintained. With its longer life cycle and more widely spaced periods of IE, E, and L protein expression, Ad may be better suited than HSV for the serial expression of transgenes.

Endogenous viral promoters can also be used to restrict transgene expression to tumor cells. Thus, if the early events of viral infection - attachment, penetration, IE, or E expression - can be restricted to tumor cells, normal cells will not support viral DNA replication and will not be lysed. Similarly, substituting a therapeutic transgene for a late gene that depends on viral DNA replication for expression should restrict transgene expression to productively infected tumor cells.

\section{Transgene delivery from replication-selective oncolytic viruses}

The use of replication-selective viruses to deliver therapeutic genes is a natural extension of current virusbased replication-defective vectors. Recombinant viruses of this type, armed therapeutic viruses (ATVs), offer several advantages for the treatment of cancer, as summarized in Figure 1. While the ATV concept is in its infancy, it represents an important evolution in virusbased cancer therapies that may find practical application in the clinical setting.

Several groups have coupled genetically engineered oncolytic viruses with a prodrug-converting enzyme (Springer and Niculescu-Duvaz, this Perspective series, ref. 15). These enzymes convert nontoxic prodrugs into cytotoxic metabolites, many of which are soluble and can act at a distance from the prodrug enzyme-expressing cell. This approach takes advantage of the tumor specificity of the virus to concentrate the prodrug-converting enzyme activity to the area of the infected tumor cell while minimizing exposure and damage to normal cells. Because many of the activated prodrugs target DNA replication either directly or indirectly, the administration schedule of the prodrug will likely need to be optimized to ensure that viral replication is maintained, if the additive effects of viral lysis and chemotherapy are to be realized. Dosing regimens that could be used to control or eradicate the viral infection should also be devised, in case other mechanisms for controlling viral infection (Martuza, this Perspective series, ref. 6) prove inadequate.

Examples of prodrug-converting enzymes expressed in replicating oncolytic human Ad include thymidine kinase (TK) and a chimeric fusion of TK and cytosine deaminase (CD) (16-18). In both cases, the constitutively active human cytomegalovirus enhancer-promoter was used to drive transgene expression from tumor-selective, $E 1 B-55 K$-deleted viruses $(7,8)$. These viruses validated a number of concepts that support the use of therapeutic transgenes in a replication-selective virus. First, viral replication and spread amplifies the expression of therapeutic protein, with up to a 2000 -fold increase in enzymatic activity relative to the replication-defective virus. Second, the dosing regimen of the prodrug significantly influences the efficacy of the treatment. Allowing viral replication to occur by administering the prodrug at day 3 instead of day 1 after infection resulted in a significant increase in animal survival and long-term cures. Finally, this replication-competent virus is indeed susceptible to prodrugactivated chemotherapy, confirming that this therapy could be used to control a viral infection.

Insertion of the "double suicide" $\mathrm{CD} / \mathrm{TK}$ fusion gene into human Ad extended the concept of creating a multimodal cancer therapy from a single agent. When fused together, these prodrug-converting enzymes were shown to be functional and superior to either single 
agent alone $(19,20)$. Interestingly, Freytag and colleagues (18) showed that double suicide gene therapy also sensitized cells to the toxic effects of radiation. Most recently, a trimodal approach (lytic virus, double suicide gene therapy, and radiation) was found to be superior to any other combination, as measured by tumor growth delay and cure rate. In this regimen, prodrug dosing was an important factor (21).

Suicide genes have also been incorporated into the replication-selective oncolytic HSV. The HSV mutant rRp450 uses the endogenous viral ICP6 promoter to drive transgene expression of the rat prodrug-converting gene CYP2B1 (22). In this work, the CYP2B1 gene was inserted into the HSV genome, replacing the HSV ribonucleotide reductase (Hsrr) gene. The deletion of Hsrr limits efficient viral replication to cancer cells (or, potentially, other cells with elevated levels of ribonucleotide reductase activity) and, hence, is responsible for tumor selectivity. CYP2B1 is a member of the cytochrome $\mathrm{P} 450$ family that converts the prodrug cyclophosphamide (CPA) into the active metabolite phosphoramide mustard. The addition of CPA had minimal effects on the productive viral infection but enhanced the antitumoral effects of the virus. This virus also retains its endogenous TK gene, another prodrug-converting enzyme whose active metabolite displays pharmacological synergy with CPA treatment (23). Interestingly, the TK activity did not show antitumor synergy with the virus alone $(23,24)$.

The immunostimulatory genes (see Agha-Mohammadi and Lotze, this Perspective series, refs. 25 and 26) IL4 and IL12 have also been introduced into replication-selective oncolytic HSVs in an attempt to couple the lytic properties of the virus to antitumor immune response of the host. In both cases, these genes were under the transcriptional control of the early-growth response- 1 promoter and their insertion eliminated both copies of the $\gamma 34.5$ genes, which are required for neurovirulence by HSV (ref. 27; see also Martuza, this Perspective series, ref. 6). Initially IL-4 was chosen, based on its ability to stimulate both macrophage and CD8 T-cell proliferation (28). More recently, Parker et al. (29) incorporated the gene for IL-12 into a replication-competent HSV in order to direct this cytokine to brain tumors. IL-12 promotes cytolytic activity of natural killer cells and cytotoxic T lymphocytes, stimulates Th1 immune responses, and blocks angiogenesis. Both viruses enhanced antitumor activity and animal survival compared with control $\gamma 34.5$-deficient viruses, and immunohistochemical analysis revealed an influx of inflammatory cells marked by $\mathrm{CD}^{+}$and $\mathrm{CD}^{+}$lymphocytes and macrophages, supporting the possibility of a role for these cytokines in tumor suppression. As was the case for the suicide gene-containing viruses, adding these immune stimulatory effects could be detrimental to the virus; optimization of expression levels and timing will likely be required to achieve maximal therapeutic benefit.

In many of the replication-selective viruses mentioned, tumor specificity is derived from the deletion of viral genes. It is probable that the deletion of any viral protein will reduce viral virulence in vivo. An interest- ing twist on gene delivery is the selective restoration of a deleted "virulence" gene in the target neoplastic cell, using a tumor-specific promoter. G207, a recombinant HSV in clinical trials, is a double mutant, with deletions of both copies of $\gamma 34.5$ and an insertional mutation in the Hsrr gene (ref. 30; see also Martuza, this Perspective series, ref. 6). To enhance the potency of a similar double mutant virus, MGH1 (31), the $\gamma 34.5$ gene was reintroduced into the viral genome under the control of the promoter from the cell cycle-regulated cellular gene $B$-myb. This virus, Myb34.5, maintained the neuroattenuation seen with other $\gamma 34.5$ deletion strains but showed improved efficacy toward the target tumor, compared with the parental double mutant virus (32). This virus represents a unique example of enhancing oncolytic potential through the controlled expression of an endogenous virulence factor.

\section{Selection of transgene insertion sites}

To date, transgenes have been inserted into areas of the viral genome that have been deleted or inactivated to confer selectivity to the viral infection. Future work may determine whether other sites might be optimal for transgene insertions. Both human Ad and HSV carry genes that are not essential for viral replication in vitro. Although these genes have been viewed as appropriate transgene insertion sites, they may well have important roles in vivo. Indeed, successful viral DNA packaging is limited by the genome size, creating a strong evolutionary pressure for the virus to retain only genes that allow for optimal viral infection and replication. For example, some of the apparently dispensable genes in the E3 region of human Ad (33-36) encode immunoregulatory proteins that allow the virus to evade immune-mediated clearance (37) and establish persistent infections (38).

Regions of the viral genome thought to be nonessential based on in vitro work will need to be carefully examined for potential roles in vivo before being used as therapeutic transgene insertion sites. Since the lytic function of the virus is crucial to maximizing antitumor efficacy, it would seem prudent to maintain immunoregulatory genes that have evolved to enable the virus to persist in the face of immune responses. Recent work demonstrating that an intact E3 region enhanced the antitumoral efficacy of a replication-selective virus appears to support this hypothesis (39). However, the E3 region includes multiple genes and might still serve as a site for transgene insertion once the genes responsible for this enhanced efficacy are better understood. In addition, whether an enhanced immune response is a desirable addition to the treatment should guide the decision to delete or to retain such immunoregulatory genes. The nature of the therapeutic transgene to be inserted, the design of the vector, and the dose and route of the recombinant virus administered will doubtless figure in this decision.

\footnotetext{
1. Verma, I.M., and Somia, N. 1997. Gene therapy: promises, problems and prospects. Nature. 389:239-242.

2. Kay, M.A., Liu D., and Hoogerbrugge, P.M. 1997. Gene therapy. Proc. Natl. Acad. Sci. USA. 94:12744-12746.

3. Anderson, W.F. 1998. Human gene therapy. Nature. 392(Suppl.):25-30.

4. Kirn, D.H., and McCormick, F. 1996. Replicating viruses as selective cancer therapeutics. Mol. Med. Today. 2:519-527.
} 
5. Heise, C., and Kirn, D.H. 2000. Replication-selective adenoviruses as oncolytic agents. J. Clin. Invest. 105:847-851.

6. Martuza, R.L. 2000. Conditionally replicating herpes vectors for cancer therapy. J. Clin. Invest. 105:841-846.

7. Bischoff, J.R., et al. 1996. An adenovirus mutant that replicates selectively in p53-deficient human tumor cells. Science. 274:373-376.

8. Heise, C., et al. 1997. ONYX-015, an E1B gene-attenuated adenovirus, causes tumor-specific cytolysis and antitumoral efficacy that can be augmented by standard chemotherapeutic agents. Nat. Med. 3:639-645.

9. Yew, P.R., and Berk, A.J. 1992. Inhibition of p53 transactivation required for transformation by adenovirus early $1 \mathrm{~B}$ protein. Nature. 357:82-85.

10. Barker, D.D., and Berk, A.J. 1987. Adenovirus proteins from both E1B reading frames are required for transformation of rodent cells by viral infection and DNA transfection. Virology. 156:107-121.

11. Matzinger, P. 1994. Tolerance, danger, and the extended family. Annu. Rev. Immunol. 12:991-1045.

12. Toda, M., Rabkin, S.D., Kojima, H., and Martuza, R.L. 1999. Herpes simplex virus as an in situ cancer vaccine for the induction of specific antitumor immunity. Hum. Gene Ther. 10:385-393.

13. Bett, A.J., Prevec, L., and Graham, F.L. 1993. Packaging capacity and stability of human adenovirus type 5 vectors. J. Virol. 67:5911-5921.

14. Rodriguez, R., et al. 1997. Prostate attenuated replication competent adenovirus (ARCA) CN706: a selective cytotoxic for prostate-specific antigen-positive prostate cancer cells. Cancer Res. 57:2559-2563.

15. Springer, C.J., and Niculescu-Duvaz, I. 2000. Prodrug-activating systems in suicide gene therapy. J. Clin. Invest. 105:1161-1167.

16. Wildner, O., Blaese, R.M., and Morris, J.C. 1999. Therapy of colon cancer with oncolytic adenovirus is enhanced by the addition of herpes simplex virus-thymidine kinase. Cancer Res. 59:410-413.

17. Wildner, O., et al. 1999. Adenoviral vectors capable of replication improve the efficacy of HSFtk/GCV suicide gene therapy of cancer. Gene Ther. 6:57-62.

18. Freytag, S.O., Rogulski, K.R., Paielli, D.L., Gilbert, J.D., and Kim, J.H 1998. A novel three-pronged approach to kill cancer cells selectively: concomitant viral, double suicide gene, and radiotherapy. Hum. Gene Ther. 9:1323-1333.

19. Rogulski, K.R., Zhang, K., Kolozsvary, A., Kim, J.H., and Freytag, S.O. 1997. Pronounced antitumor effects and tumor radiosensitazation of double suicide gene therapy. Clin. Cancer Res. 3:2081-2088

20. Rogulski, K.R., Kim, J.H., Kim, S.H., and Freytag, S.O. 1997. Gilioma cells transduced with an Escherichia coli CD/HSV-1 TK fusion gene exhibit enhanced metabolic suicide and radiosensitivity. Hum. Gene Ther. 8:73-85.

21. Rogulski, K.R., et al. 2000. Double suicide gene therapy augments the antitumor activity of a replication-competent lytic adenovirus through enhanced cytotoxicity and radiosensitization. Hum. Gene Ther. 11:67-76.

22. Chase, M., Chung, R.Y., and Chiocca, E.A. 1998. An oncolytic viral mutant that delivers the CYP2B1 transgene and augments cyclophosphamide chemotherapy. Nat. Biotechnol. 16:444-448.

23. Aghi, M., Chou, T.C., Sulling, K., Breakfield, X.O., and Chiocca, E.A.
1999. Multimodal cancer treatment mediated by a replicating oncolytic virus that delivers the oxazaphosphorine/rat cytochrome P450 2B1 and ganciclovir/herpes simplex virus thymidine kinase gene therapies. Cancer Res. 59:3861-3865

24. Yoon, S.S., Carroll, N.M., Chiocca, E.A., and Tanabe, K.K. 1998. Cancer gene therapy using a replication-competent herpes simplex virus type 1 vector. Ann. Surg. 228:366-374.

25. Agha-Mohammadi, S., and Lotze, M.T. 2000. Immunomodulation of cancer: potential use of selectively replicating agents. J. Clin. Invest. 105:1173-1176.

26. Agha-Mohammadi, S., and Lotze, M.T. 2000. Regulatable systems: applications in gene therapy and replicating viruses. J. Clin. Invest. 105:1177-1183.

27. Chou, J., Kern, E.R., Whitley, R.J., Roizman, B. 1990. Mapping of herpes simplex virus-1 neurovirulence to gamma 134.5, a gene nonessential for growth in culture. Science. 250:1262-1266.

28. Andreansky, S., et al. 1998. Treatment of intracranial gliomas in immunocompetent mice using herpes simplex viruses that express murine interleukins. Gene Ther. 5:121-130.

29. Parker, J.N., et al. 2000.Engineered herpes simplex virus expressing IL12 in the treatment of experimental murine brain tumors. Proc. Natl. Acad. Sci. USA. 97:2208-2213.

30. Mineta, T., Rabkin, S.D., Yazaki, T., Hunter, W.D., and Martuza, R.L. 1995. Attenuated multi-mutated herpes simplex virus1 for the treatment of malignant gliomas. Nat. Med. 1:938-943.

31. Kramm, C.M., et al. 1997 Therapeutic efficiency and safety of a secondgeneration replication-conditional HSV1 vector for brain tumor gene therapy. Hum. Gene Ther. 8:2057-2068.

32. Chung, R.Y., Saeki, Y., and Chiocca, E.A. 1999. B-myb promoter retargeting of herpes simplex virus gamma34.5 gene-mediated virulence toward tumor and cycling cells. J. Virol. 73:7556-7564.

33. Berkner, K.L., and Sharp, P.A. 1983. Generation of adenovirus by transfection of plasmids. Nucleic Acids Res. 11:6003-6020.

34. Kelly, T.J., and Lewis, A.M., Jr. 1973. Use of nondefective adenovirus-simian virus 40 hybrids for mapping the simian virus 40 genome. J. Virol. 12:643-652.

35. Flint, S.J., Wewerka-Lutz, Y., Levine, A.S., Sambrook, J., and Sharp, P.A. 1975. Adenovirus transcription. II. RNA sequences complementary to simian virus 40 and adenovirus 2DNA in AD2+ND1- and AD2+ND3infected cells. J. Virol. 16:662-673.

36. Jones, N., and Shenk, T. 1978. Isolation of deletion and substitution mutants of adenovirus type 5. Cell. 13:181-188.

37. Wold, W.S., et al. 1999. Immune responses to adenoviruses: viral evasion mechanisms and their implications for the clinic. Curr. Opin. Immunol. 11:380-386.

38. Fox, J.P., Hall, C.E., and Cooney, M.K. 1977. The Seattle Virus Watch. VII. Observations of adenovirus infections. Am. J. Epidemiol. 105:362-386.

39. Yu, D.C., Chen, Y., Seng, M., Dilley, J., and Henderson, D.R. 1999. The addition of adenovirus type 5 region E3 enables calydon virus 787 to eliminate distant prostate tumor xenografts. Cancer Res. 59:4200-4203. 